\title{
Orthotopic Heart Transplant: A Therapeutic Option for Unresectable Cardiac Fibroma in Infants
}

\author{
Daisuke Kobayashi, MD, Thomas J. L'Ecuyer, MD, and Sanjeev Aggarwal, MD \\ Division of Pediatric Cardiology, Carman and Ann Adams Department of Pediatrics, Children's Hospital of Michigan, \\ Wayne State University School of Medicine, Detroit, Mich, USA
}

\begin{abstract}
A B S T R A C T
Primary cardiac tumors are rare lesions in childhood, with the two most common being rhabdomyoma and fibroma. We report two infants who successfully underwent orthotopic heart transplant for massive interventricular septal cardiac fibromas. For unresectable infantile cardiac fibroma, orthotopic heart transplant may be considered a therapeutic option.
\end{abstract}

Key Words. Cardiac Fibroma; Infant; Orthotopic Heart Transplant

\section{Introduction}

$\mathrm{F}$ ibroma is the second most common cardiac tumor after rhabdomyoma in children. ${ }^{1}$ Cardiac fibroma is usually a large, solitary, firm, nonencapsulated tumor that arises from the left ventricular free wall or septum and tends not to regress spontaneously. ${ }^{1}$ Complete surgical resection has been reported with an excellent outcome. ${ }^{2}$ In certain cases, however, the tumor may be unresectable because of potential risk to vital cardiac structures. ${ }^{3}$ We report two infants who successfully underwent orthotopic heart transplantation $(\mathrm{OHT})$ for massive interventricular septal cardiac fibromas.

\section{Case Reports}

Case I

A fetal echocardiogram obtained in a 44-year-old G11P6 mother at 30 weeks gestation revealed a large solitary mass occupying most of the fetal left ventricle. There was no obstruction to left ventricular inflow or outflow. A repeat fetal echocardiogram at 31 weeks gestation revealed an enlarging mass with no signs of congestive heart failure (Figure 1A). The infant was born at 33 weeks gestation via emergent Cesarean section

(C) 2011 Wiley Periodicals, Inc. because of chorioamnionitis, with a birth weight of $2135 \mathrm{~g}$. The Apgar scores were six and eight at 1 and 5 minutes, respectively. At birth, she was asymptomatic and had no dysmorphic features. The postnatal echocardiogram confirmed a solitary mass arising from the interventricular septum and occupying most of the left ventricular cavity without restriction to flow (Figure 1B). Mild to moderate mitral insufficiency was noted without pericardial effusion. A cardiac magnetic resonance imaging (MRI) confirmed that the mass arising from the interventricular septum measured $4.0 \times$ $2.0 \mathrm{~cm}$ and occupied most of the left ventricular cavity (Figure 2). Brain MRI and abdominal ultrasound were unremarkable. She remained asymptomatic until 2 months of age, when her enlarging tumor caused respiratory distress that is characterized by tachypnea and mild retractions. Repeat echocardiography showed an interval increase in tumor size with increased internal heterogeneity (Figure 1C). Repeat MRI revealed that the mass measuring $4.8 \times 4.5 \times 4.0 \mathrm{~cm}$ was homogeneously isointense on T1 sequences and hypointense on gradient-echo sequences (Figure 2). There was heterogeneous peripheral contrast enhancement with areas of central nodular enhancement within the mass lesion. Cardiac catheterization was performed to assess hemodynamics. During

Congenit Heart Dis. 2012;7:E31-E36 


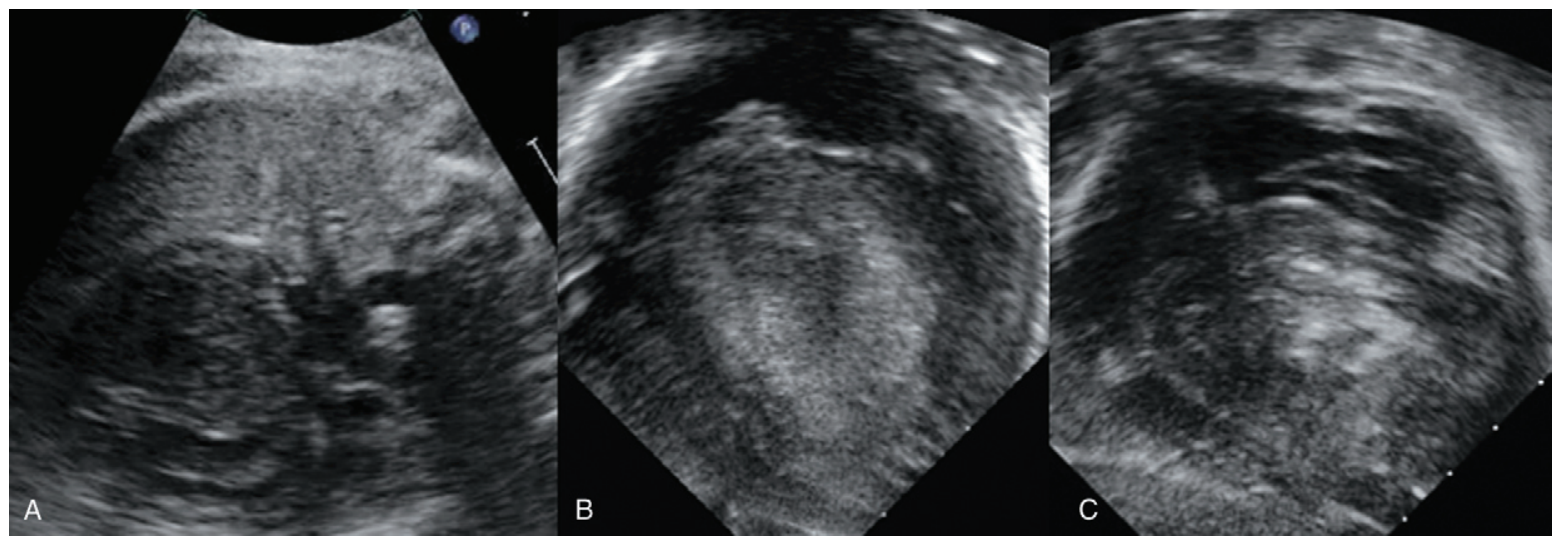

Figure 1. Case 1. (A) Four-chamber view on fetal echocardiography at 31 weeks gestation, showing the large interventricular mass occupying the left ventricle. (B) Four-chamber view at 1 day of age, showing a solitary mass arising from the interventricular septum. (C) Four-chamber view at 2 month of age, showing increased internal heterogenicity.

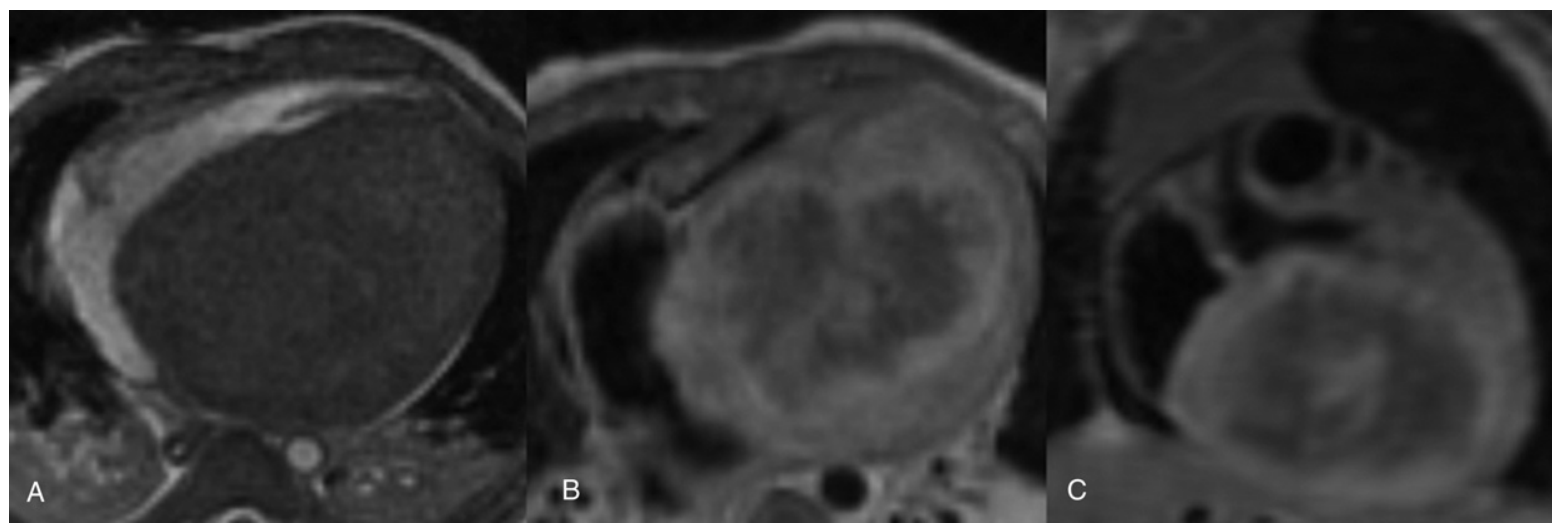

Figure 2. Case 1. Cardiac MRI. (A) Four-chamber view, showing that the mass was homogeneously isointense on T1 sequences. (B) Four-chamber view with contrast, showing heterogeneous peripheral enhancement with areas of central nodular enhancement within the lesion. (C) Coronal view with contrast.

catheterization, the tumor was thought to be approachable by biopsy catheter, and as the possibility of malignancy was not entirely excluded in the rapidly growing tumor, a diagnostic endomyocardial biopsy was performed. Systolic right ventricular pressure was two-thirds of systemic with a peak gradient of $20 \mathrm{~mm} \mathrm{Hg}$ across the right ventricular outflow tract. The mean left atrial pressure was $7 \mathrm{~mm} \mathrm{Hg}$ with a-wave of $11 \mathrm{~mm} \mathrm{Hg}$. The end-diastolic left ventricular pressure was $4 \mathrm{~mm} \mathrm{Hg}$. The pulmonary vascular resistance was 2.3 indexed Woods unit. This slight increase in the pulmonary vascular resistance was not a contraindication for OHT. There was no gradient across the left ventricular outflow tract. Endomyocardial biopsy was not diagnostic. An open biopsy was obtained at 4 months of age, which suggested cardiac fibroma. Following the biopsy, the infant had difficulty weaning off the ventilator; bronchoscopy revealed compression of the left bronchus. The tumor was deemed to be unresectable, therefore the infant was listed for OHT and received an organ after 3 months. She remained intubated for the entire period. Macroscopic examination of the explanted heart revealed a well-circumscribed mass measuring $6.8 \times 4.8 \times 4.6 \mathrm{~cm}$ located within the interventricular septum and posterior wall (Figure 3). The cut surface of the mass was firm pink-tan with fine trabeculation. Microscopic examination confirmed a cardiac fibroma that is characterized by fibroblasts admixed with scattered collagen bundles. Cytogenetic testing of fibroblasts revealed 46XX, del(9)(q21.2q34) in all the 20 cultured metaphase cells. Following her $\mathrm{OHT}$, she required prolonged intubation but was eventually extubated after 36 days and was dis- 


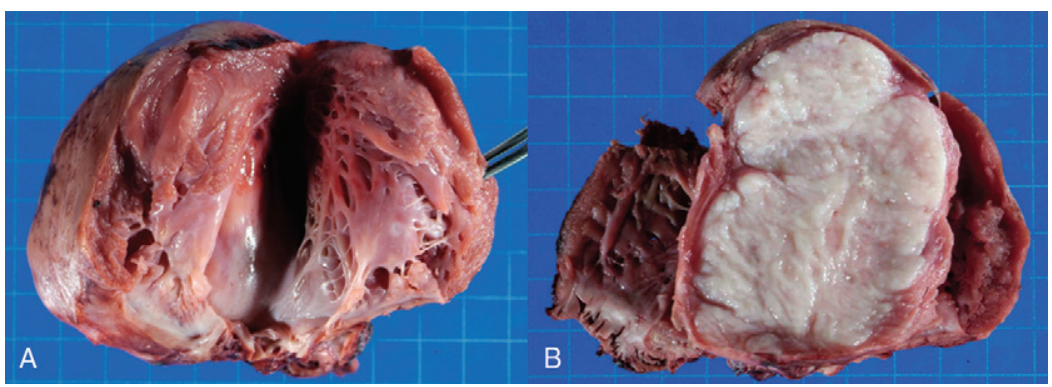

Figure 3. Case 1. (A) Macroscopic examination of the explanted heart, showing a well-circumscribed mass measuring 6.8 $\times 4.8 \times 4.6 \mathrm{~cm}$ located within the interventricular septum and posterior wall. (B) The cut surface of the mass was firm pink-tan with fine trabeculation.

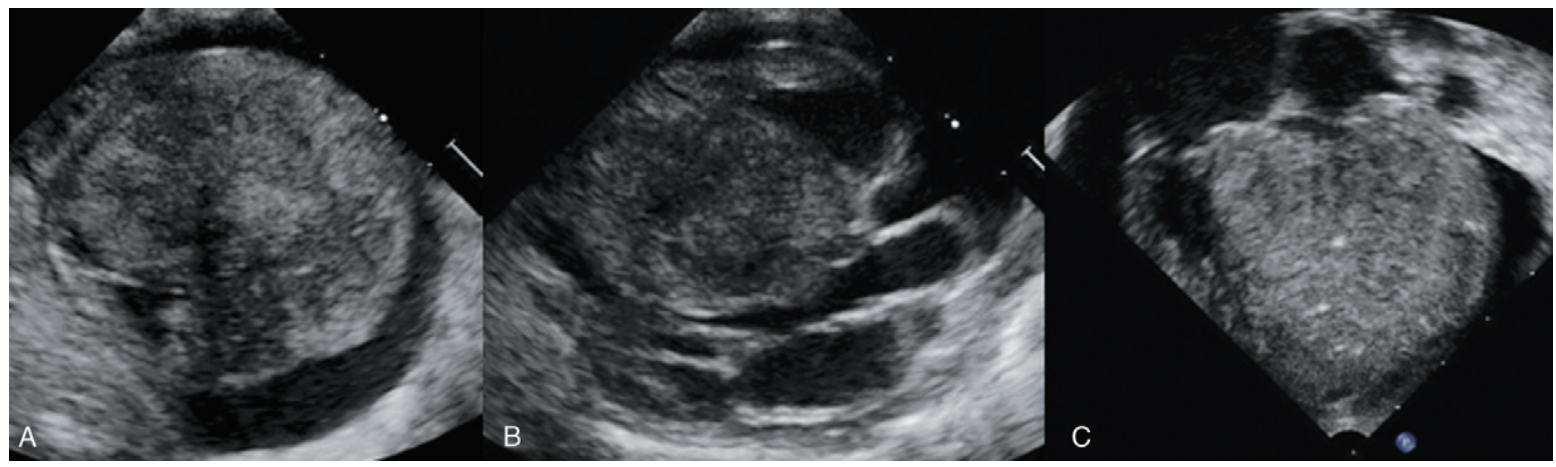

Figure 4. Case 2. Echocardiography at 1 day of age. (A) Short-axis view, showing a large mass occupying most of the left ventricle as well as a moderate pericardial effusion. (B) Long-axis view, showing a mass arising from the interventricular septum. (C) Four-chamber view, showing the interventricular mass, occupying both of the ventricles.

charged after 46 days. At the latest follow-up after 3 years, she remains asymptomatic and has had no return of any intracardiac mass or other tumors.

\section{Case 2}

This infant was born to a 27-year-old G3P2 mother at 40 weeks gestation via Cesarean section. Prenatal history was unremarkable. She required bag and mask ventilation at delivery. The Apgar scores were two and nine at 1 and 5 minutes, respectively. She was large for gestational age, with a birth weight of $3991 \mathrm{~g}$. A chest x-ray performed for respiratory distress revealed cardiomegaly. Karyotype was 46XX. Echocardiogram showed a large mass in the interventricular septum occupying much of the left ventricular cavity and impinging on the left ventricular outflow tract (Figure 4). A moderate pericardial effusion was treated with pericardiocentesis. Cytology of pericardial fluid showed numerous neutrophils and monocytes without malignant cells. Cardiac MRI confirmed that the mass arose from the interventricular septum, occupied the superior left ventricle, and measured $4.7 \times 4.5 \times 2.6 \mathrm{~cm}$ (Figure 5). The mass was homogeneously isointense on $\mathrm{T} 1$ sequences and mildly hyperintense on T2 sequences. There was irregular peripheral contrast enhancement that extended into the free wall of the left ventricle, suggesting that the tumor was invading the left ventricular wall. Brain and abdominal MRI were unremarkable. The tumor appeared unresectable because of its size, location, and permeation of the septum, therefore, she was listed for OHT. She was discharged home at 3 weeks of age, with nasogastric tube feeding for poor weight gain. Over the next 6 months, she required several hospitalizations because of respiratory distress and failure to thrive. Serial echocardiography showed a persistent large cardiac tumor. At 4 months of age, she developed severe respiratory distress and required emergent intubation because of complete collapse of the left mainstem bronchus on bronchoscopy. She required inotropic support for impaired cardiac function. A successful OHT was performed at 6 months of age. Macroscopic examination showed a large 


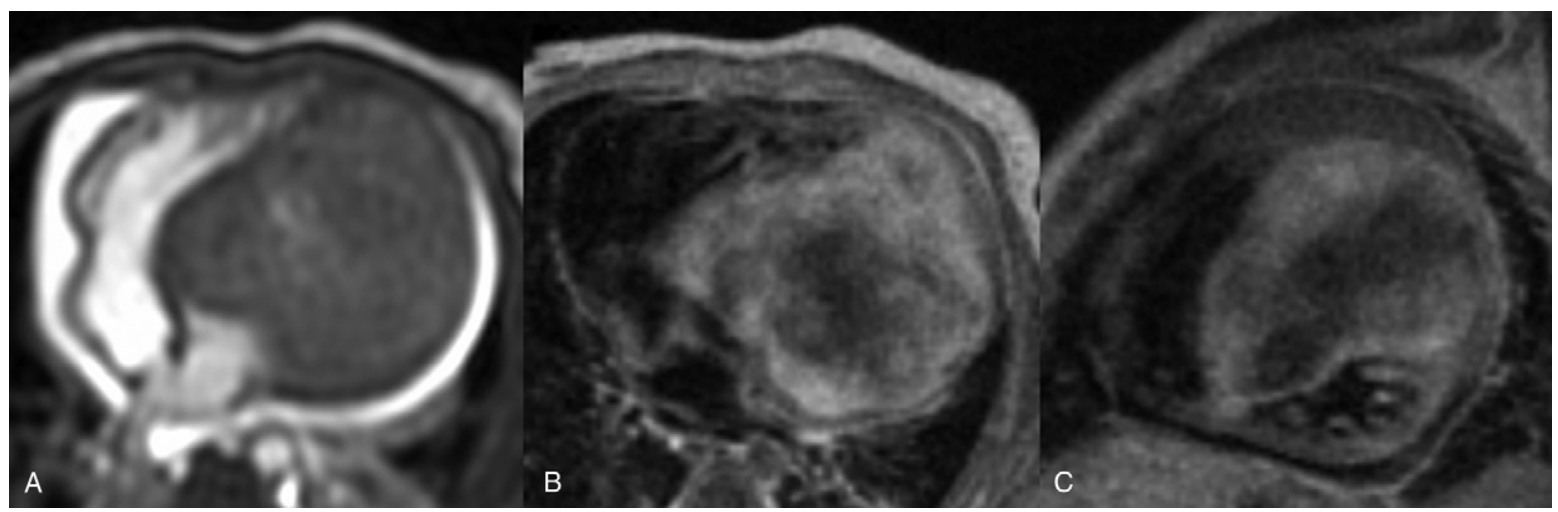

Figure 5. Case 2. Cardiac MRI. (A) Four-chamber view, showing that the mass was homogeneously isointense on $\mathrm{T} 1$ sequences. (B) Four-chamber view with contrast, showing heterogeneous peripheral enhancement. (C) Axial view with contrast.

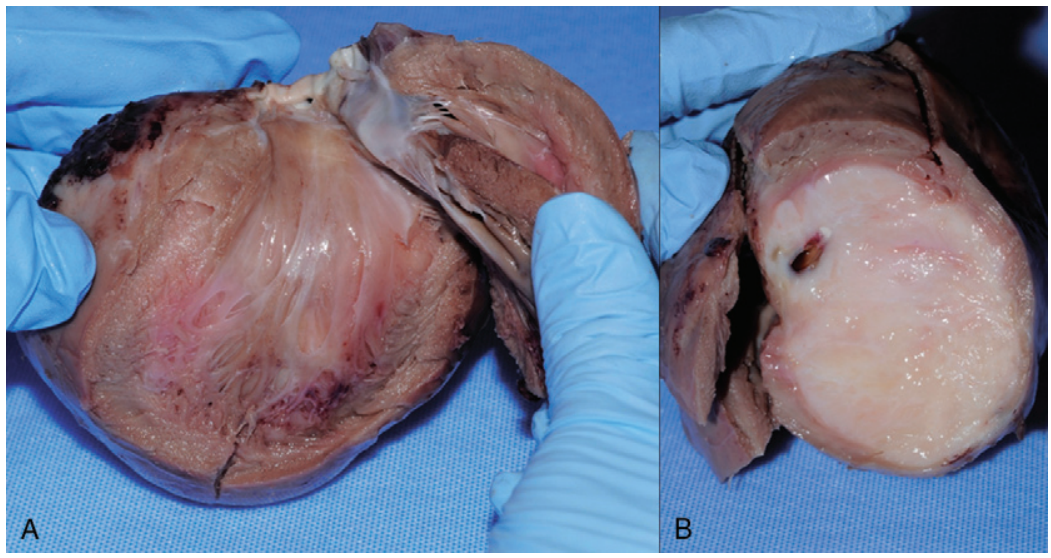

Figure 6. Case 2. (A) Macroscopic examination of the explanted heart, showing a well-circumscribed mass measuring $6 \times$ $4 \times 7 \mathrm{~cm}$ located within the interventricular septum. (B) The cut surface of the mass was firm gray-white, with a focal small cleft-like cystic area measuring $0.6 \mathrm{~cm}$ in diameter.

solid gray-white tumor measuring $6 \times 4 \times 7 \mathrm{~cm}$, which arose from the interventricular septum and compressed the cardiac conducting bundles (Figure 6). The tumor occupied the entire cavity of both ventricles. There was a focal small cleftlike cystic area measuring $0.6 \mathrm{~cm}$ in diameter. Microscopic examination confirmed a cardiac fibroma. Cytogenetic testing of fibroblasts was unsuccessful. Following her OHT, she was successfully extubated after 22 days and was discharged after 34 days. She remains asymptomatic at her 19 months of follow-up and has had no recurrence or other tumors.

\section{Discussion}

Primary cardiac tumors are rare lesions with a reported incidence of $<0.1 \% .^{1}$ Among the pediat- ric cardiac tumor, rhabdomyoma is the most common; fibroma is the second most common with more than $80 \%$ of cardiac fibromas occurring in children. ${ }^{4}$ Cardiac fibroma often involves the left ventricular free wall or septum and less frequently involves the right ventricle and atria. ${ }^{1}$ Microscopic features include the presence of fibroblasts with no encapsulation with infiltration of the tumor at the junctional areas between the tumor and the normal tissues. ${ }^{4}$ Focal calcification is frequent with occasional cystic degeneration. ${ }^{1}$ Although cardiac fibromas are considered histologically benign, they can manifest as arrhythmias $^{5,6}$ and sudden death ${ }^{7}$ and lead to congestive heart failure secondary to hemodynamic obstruction. In our two cases, the cardiac fibroma caused bronchial compression and respiratory distress requiring intubation. 
Symptomatic fibromas have been successfully treated with partial or complete resection. ${ }^{2,8}$ When complete resection is not possible, partial resection or "debulking" can help to restore normal hemodynamic and respiratory function. ${ }^{9,10}$ A palliative approach has been recently reported in infants with hemodynamic obstruction because of cardiac fibroma. ${ }^{11,12}$ An infant with a large right ventricular fibroma underwent a Blalock-Taussing shunt as initial palliation and subsequently completed resection of the tumor at 9 years of age. ${ }^{11}$ Freire et al. reported that two neonates with large left ventricular fibroma underwent the hybrid procedure (bilateral pulmonary artery banding, ductal stenting, followed by balloon atrial septostomy), resulting in univenricular physiology. ${ }^{12}$ In our two cases, the tumors were deemed to be unresectable because of their location, aggressive growth, difficulty in distinguishing them from myocardium, and risk to conduction pathways. In addition, debulking and other palliative options were considered but were thought to be unlikely to relieve bronchial compression and posed risks of continued growth and arrhythmias. Therefore, OHT was selected as definitive therapy. OHT has been performed in unresectable primary cardiac tumors. ${ }^{3}$ Scattered case reports have previously described successful OHT in infants with cardiac fibroma. ${ }^{13-15}$

An echocardiogram remains the initial diagnostic noninvasive modality to evaluate cardiac function and hemodynamic disturbance secondary to tumor. Cardiac MRI is an excellent imaging modality to delineate the extent and size of the cardiac tumor and to detect compression on contiguous extracardiac structures. Cardiac fibroma appears as a low signal on T1-weighted images and homogeneously hypointense or low signal on T2-weighted sequences while being relatively hypovascular on first-pass perfusion imaging and homogeneous, peripheral, or heterogeneous on delayed enhancement. ${ }^{16}$ Rhabdomyomas, on the other hand, are isointense to marginally hyperintense to myocardium on T1-weighted images and hyperintense on T2-weighted images while being hypointense to myocardium after contrast administration. ${ }^{17}$ Gadolinium contrast administration is useful for tissue characterization and improves mass delineation. Heterogeneous delayed enhancement suggests regional variations in vascularity and distribution volumes such as seen with areas of necrosis within a tumor. Hoffmann et al. reported on the usefulness of cardiac MRI to distinguish malignant from benign cardiac and paracardiac masses in 55 patients. ${ }^{18}$ The predictors for malignant cardiac or paracardiac masses were right-sided cardiac location, inhomogeneity of tumor tissue, and presence of effusion. Pediatric data on the utility of MRI in tumors are scarce. Kiaffas et al. described cardiac MRI characteristics in 11 infants and children with primary cardiac tumors. ${ }^{19}$ It has been suggested that cardiac MRI is useful in children with cardiac tumors in whom the diagnosis of rhabdomyoma is considered unlikely because rhabdomyoma is usually easily diagnosed by echocardiography. A T2-weighted spin echo sequence helps to distinguish vascular tumors, such as hemangiomas from avascular tumors such as fibroma. Although there is no pathognomic finding in cardiac MRI, it can provide important diagnostic information about tumor tissue characteristics, anatomy, and hemodynamic consequences.

Cardiac fibroma occurs sporadically or as a feature of Gorlin syndrome, a rare autosomal dominant disorder characterized by basal cell carcinoma, craniofacial, central nervous system, musculoskeletal, and genitourinary abnormalities. ${ }^{20}$ Gorlin syndrome is caused by mutations in the PTCH1 that is located in $9 \mathrm{q} 22.3$ and acts as a tumor suppressor gene. ${ }^{20}$ Scanlan et al. recently reported homozygous or heterozygous loss of the PTCH1 locus that was identified in three nonsyndromic cardiac fibromas. ${ }^{21}$ There are two other cardiac fibromas reporting genetic rearrangements of $9 \mathrm{q} 22$ at $(1 ; 9)(\mathrm{q} 32)^{22}$ and at $(1 ; 9 ; 5)\left(\mathrm{q} 24\right.$ 'q22;q22). ${ }^{23}$ In our case 1 , cytogenetic testing of fibroblasts revealed abnormal metaphases having a partial/interstitial deletion in the deletion of chromosome $9 \mathrm{q} 21.2 \mathrm{q} 34$ as a clonal abnormality.

In summary, we report two infants with unresectable interventricular septal cardiac fibroma who successfully underwent OHT in a contemporary era when OHT outcomes are much improved. A large cardiac fibroma can cause bronchial compression, requiring mechanical ventilation. In case surgical resection is not feasible, OHT may be considered as an option of treatment.

Author Contributions: All the three authors drafted and critically revised the manuscript.

Institution where work was done: Children's Hospital of Michigan, Detroit, Michigan.

Corresponding Author: Daisuke Kobayashi, MD, Division of Pediatric Cardiology, Children's Hospital of Michigan, Carman and Ann Adams Department of 
Pediatrics, Wayne State University School of Medicine, 3901 Beaubien Blvd, Detroit, MI 48201-2119, USA. Tel: (+1313) 745-5993; Fax: (+1313) 993-0894; E-mail: dkobayas@dmc.org

Conflict of interest: No conflict of interest, grants, or other financial support.

Accepted in final form: August 3, 2011.

\section{References}

1 Freedom RM, Lee KJ, MacDonald C, Taylor G. Selected aspects of cardiac tumors in infancy and childhood. Pediatr Cardiol. 2000;21:299-316.

2 Cho JM, Danielson GK, Puga FJ, et al. Surgical resection of ventricular cardiac fibromas: early and late results. Ann Thorac Surg. 2003;76:1929-1934.

3 Gowdamarajan A, Michler RE. Therapy for primary cardiac tumors: is there a role for heart transplantation? Curr Opin Cardiol. 2000;15:121-125.

4 Gotlieb AI. Cardiac fibromas. Semin Diagn Pathol. 2008;25:17-19.

5 Stratemann S, Dzurik Y, Fish F, Parra D. Left ventricular cardiac fibroma in a child presenting with ventricular tachycardia. Pediatr Cardiol. 2008;29: 223-226.

6 Sharma K, Rohlicek C, Cecere R, Tchervenkov CI. Malignant arrhythmias secondary to a cardiac fibroma requiring transplantation in a teenager. 7 Heart Lung Transplant. 2007;26:639-641.

7 Meissner C, Minnasch P, Gafumbegete E, Reiter A, Gerling I, Oehmichen M. Sudden unexpected infant death due to fibroma of the heart. 7 Forensic Sci. 2000;45:731-733.

8 Stiller B, Hetzer R, Meyer R, et al. Primary cardiac tumours: when is surgery necessary? Eur $\mathcal{F}$ Cardiothorac Surg. 2001;20:1002-1006.

9 D'hondt B, Rega F, Mertens L, Gewillig M, Meyns B. Primary cardiac fibroma: a rising giant in a small cavity-size does matter. Heart Surg Forum. 2008;11:E134-E136.

10 Usta E, Hofbeck M, Kaulitz R, Sieverding L, Greil G, Ziemer G. Giant infantile fibroma of the right ventricle-surgical debulking and tumor plication. Thorac Cardiovasc Surg. 2009;57:304-305.
11 Lee C, Kim SJ, Kim YM. Cardiac fibroma in an infant: complete resection after a Blalock-Taussig shunt as initial palliation. Ann Thorac Surg. 2010;90: 1011-1014.

12 Freire G, Jureidini S, Schowengerdt KO, Rahimi B, Tinker K, Fiore AC. Hybrid procedure for obstructive neonatal left ventricular tumors. Ann Thorac Surg. 2010;90:2057-2059.

13 Dunn JM, Cavarocchi NC, Balsara RK, et al. Pediatric heart transplantation at St. Christopher's Hospital for Children. 7 Heart Transplant. 1987;6: 334-342.

14 Michler RE, Goldstein DJ. Treatment of cardiac tumors by orthotopic cardiac transplantation. Semin Oncol. 1997;24:534-539.

15 Jamieson SW, Gaudiani VA, Reitz BA, Oyer PE, Stinson EB, Shumway NE. Operative treatment of an unresectable tumor of the left ventricle. 7 Thorac Cardiovasc Surg. 1981;81:797-799.

16 Hoey ET, Mankad K, Puppala S, Gopalan D, Sivananthan MU. MRI and CT appearances of cardiac tumours in adults. Clin Radiol. 2009;64: 1214-1230.

17 Sparrow PJ, Kurian JB, Jones TR, Sivananthan MU. MR imaging of cardiac tumors. Radiographics. 2005;25:1255-1276.

18 Hoffmann U, Globits S, Schima W, et al. Usefulness of magnetic resonance imaging of cardiac and paracardiac masses. Am 7 Cardiol. 2003;92:890-895.

19 Kiaffas MG, Powell AJ, Geva T. Magnetic resonance imaging evaluation of cardiac tumor characteristics in infants and children. Am 7 Cardiol. 2002;89:1229-1233.

20 Gorlin RJ. Nevoid basal cell carcinoma (Gorlin) syndrome. Genet Med. 2004;6:530-539.

21 Scanlan D, Radio SJ, Nelson M, et al. Loss of the PTCH1 gene locus in cardiac fibroma. Cardiovasc Pathol. 2008;17:93-97.

22 Viswanathan S, Gibbs JL, Roberts P. Clonal translocation in a cardiac fibroma presenting with incessant ventricular tachycardia in childhood. Cardiol Young. 2003;13:101-102.

23 Bossert T, Walther T, Vondrys D, Gummert JF, Kostelka M, Mohr FW. Cardiac fibroma as an inherited manifestation of nevoid basal-cell carcinoma syndrome. Tex Heart Inst 7. 2006;33:88-90. 